\title{
Chitosan-Hydroxyapatite Scaffold for Tissue Engineering in Experimental Lumbar Laminectomy and Posterolateral Spinal Fusion in Wistar Rats
}

\author{
Martin Rodríguez-Vázquez, Rodrigo Ramos-Zúñiga \\ Department of Neurosciences, Translational Institute of Neuroscience, University Center of Health Sciences, \\ University of Guadalajara, Guadalajara, Mexico
}

Study Design: Experimental study with an animal model.

Purpose: To evaluate the role of a chitosan and hydroxyapatite composite for spinal fusion in a lumbar experimental model based on regenerative tissue engineering principles.

Overview of Literature: Chitosan and hydroxyapatite represent an alternative biodegradable implant material for tissue engineering and regeneration. The combination of chitosan and hydroxyapatite in a 20:80 ratio could potentiate their individual properties as an implantable composite for experimental laminectomy.

Methods: Phase I: design and synthesis of a porous composite scaffold composed of chitosan-hydroxyapatite using a freeze drying technique. Phase II: experimental microsurgical lumbar laminectomy at L5. A total of 35 Wistar rats were categorized into three experimental groups: control (laminectomy alone), experimental (laminectomy with implant), and reference (intact spine) ( $n=5$ per group). Postoperative structural and functional evaluations were performed using computed tomography scans. In addition, radiologic, clinical, histological, and immunohistochemical microstructures were evaluated.

Results: At the laminectomy site, the composite implant induced bone regeneration, which was observed in the axial reconstruction of the rat lumbar spine in all cases. Biomechanical changes in the lumbar spine were observed by radiology in both groups after the surgery. The posterolateral space was covered by a bone structure in the treated spine, a condition not seen in the control group. The range of motion was $7.662^{\circ} \pm 0.81^{\circ}$ in the scaffold group versus $20.72^{\circ} \pm 3.47^{\circ}$ in the control group. Histological findings revealed qualitatively more bone tissue formation in the implant group.

Conclusions: A composite of chitosan-hydroxyapatite at a 20:80 ratio induced bone formation after experimental laminectomy in rats and led to spinal fusion, which was assessed by radiology and biomechanical tests. No functional complications in posture or walking were observed at 90 days post-surgery, despite biomechanical changes in the spine.

Keywords: Chitosan; Hydroxyapatites; Laminectomy; Spinal fusion; Spine surgery; Tissue engineering

\section{Introduction}

Lumbar laminectomy using various modalities is a stan- dard procedure for patients with low back pain for whom conservative treatment fails. This intervention includes the release of pressure on nerve roots and spinal nerves to

Received Mar 28, 2019; Revised Jun 29, 2019; Accepted Jul 4, 2019

Corresponding author: Rodrigo Ramos-Zúñiga

Department of Neuroscience, Translational Institute of Neuroscience, University Center of Health Sciences, University of Guadalajara, Sierra Mojada 950, Col. Independencia, CP 44340, Guadalajara, Mexico

Tel: +52-33-1058-5200(33675), E-mail: rodrigor@cencar.udg.mx, rodrigorz13@gmail.com 
relieve pain [1]. Although lumbar laminectomy is still the most common procedure, it can cause spinal instability, and in many cases, an additional surgical procedure with consequent spinal fusion are needed [2]. This additional procedure involves an arthrodesis and uses rods and bars to provide spine stability [3-5]. For the purpose of spinal fusion, autologous bone grafting is required, and the iliac crest is the gold standard donor site [6]. Harvest of autologous bone grafts has been associated with postoperative infection, fractures, pain, and an extension of hospital stay [7]; therefore, bone graft substitutes are an important alternative in spinal fusion.

New materials have been developed and tested that can substitute for the autologous bone grafts used in lumbar laminectomy and spinal fusion [8]. These natural and synthetic biomaterials have desirable properties such as osteoconduction, osteoinduction, immune system tolerance, and biomechanical support.

Chitosan $(\mathrm{Ch})$ is a biomaterial that has been used as a hemostatic agent and for wound healing [9]. Its chemical composition and physical properties allow $\mathrm{Ch}$ to combine with other biomaterials or drugs, which potentiates its properties, which include degradation velocity and sustained drug release [10], as well as its mechanical and elastic properties [11].

Hydroxyapatite (HAp) has been used as a ceramic biomaterial because of its similarities to bone tissue. In addition, it provides an adequate substrate for the ingrowth of tissue during bone regeneration [12]. HAp is a brittle ceramic, and therefore, cannot be molded for use in specific bone defects. The design, percentage of concentration and association with other compounds determine its biomechanical behavior and its ability to integrate into bone, resulting in a heterogeneous pattern of absorption and degradation and variations in the degree of the bony bridge.

Currently we recognize various patterns of degradation and regenerative capacity according to the structural design of the scaffold and its tendency to develop compounds associated with bioactive elements $[13,14]$. The combination of $\mathrm{Ch}$ and HAp has gained interest in bone tissue engineering $[14,15]$ because of the characteristics shared by both: the elastic properties and compressive resistance of chitosan and the capacity for osteoinduction by Hap [16].

In the present study, we propose the synthesis and use of a new biodegradable composite of Ch/HAp at a 20:80 ratio based on a specific design for lumbar laminectomy reconstruction and spinal fusion.

\section{Materials and Methods}

\section{Biodegradable composite synthesis}

A biodegradable composite made of Ch/HAp was generated by a simple molding method, in which the final compound consisted of $2 \%$ chitosan gel (Sigma-Aldrich, Toluca, Mexico) in 2\% acetic acid with the addition of a HAp micro-powder (Sigma-Aldrich). The gelatin composite of $\mathrm{Ch} / \mathrm{HAp}$ at a 20:80 weight/volume ratio was molded in petri dishes until a desired thickness and homogenization were reached; the composite membrane was then freezedried at $4^{\circ} \mathrm{C}$ and lyophilized for 24 hours. Subsequently, a three-dimensional membrane was obtained, which was then precipitated in $1 \mathrm{~N} \mathrm{NaOH}$ for 2 hours, washed in distilled water, and finally sterilized in ethylene oxide gas. We performed a control test to verify that the biomaterial was intact in terms of its structural design after the sterilization process.

\section{Surgical procedure}

A total of 30 male Wistar rats were maintained according to the bioethics committee of the University Center of Health Sciences, Mexican Official Standard (NOM062ZOO-1999) for laboratory animal use in research, with the registration number 063-2014. Additionally, five Wistar rats with the same weight were used as a reference group (intact group) for biomechanical testing. Animals weighing 350-400 g were placed in an experimentally controlled environment with a 12:12-hour light:dark period and had ad libitum access to food and water.

For anesthesia, a mixture of ketamine:xylazine (PISA, Guadalajara, Mexico) at a dose of $40: 10 \mathrm{mg} / \mathrm{kg}$ of corporal weight was intraperitoneally administered. An incision was made in the middle line of the lumbar spine, and the iliac crest was used as a reference to localize the spinous process of the L5 vertebrae. The paravertebral muscles were then dissected, the spinous process was removed, and a drill machine (Lynx; M.T.I. Dental, Coatesville, PA, USA) with a diamond tip was used to eliminate the dorsal lamina, which was removed carefully to avoid nerve injury. Facet joints and transverse processes were also drilled and decorticated (postero-lateral extension) to generate a 
bone contact surface. Once the lamina was removed, the site was cleaned with saline and filled with a wet composite membrane covering the entire bone surgical defect and included facet joints on both ends. Finally, the muscles and fascia were sutured with vicryl 3-0, whereas the skin was sutured with nylon 3-0 (Ethicon; Johnson and Johnson, Cincinnati, OH, USA). All study animals were classified into three groups: (1) intact group $(n=5$, only for range of motion $[\mathrm{ROM}]$ reference data), (2) laminectomy with implant group $(\mathrm{n}=15)$, and (3) laminectomy alone, which served as a control group $(\mathrm{n}=15)$. Only basic control groups were considered in this study to assess biocompatibility and regenerative capacity as a first step.

\section{Radiographic dynamic evaluations}

Flexion and extension of the lumbar spine were induced with a device consisting of two acrylic plaques with a $110^{\circ}$ concave space in which, post-mortem, the extracted lumbar spines were placed and submitted to X-ray. Finally, radiographic evaluation of the ROM (difference between the flexion and extension degrees) was performed using the RadiAnt DICOM viewer software ver. 2.2.9 (Medixant, Poznan, Poland).

\section{Assessment of lumbar fusion}

Radiological and computed tomography examinations (Somatom Definition AS; Siemens Healthcare, Erlangen, Germany) of the extracted rat spine were performed, and the fusion rate was evaluated by three blinded independent observers, in accordance with the method described by Lenke et al. [17]; the specimens were then scored according to the radiographic spinal fusion examination. For manual palpation, the lumbar spine was extracted from rats sacrificed 90 days after the surgery according to the previously described method by Dimar et al. [18] in which the following values were assigned: (1) solid (fusion), (2) non-solid (non-fusion), and (3) low-motion non-solid (pseudoarthrosis).

\section{Biomechanical testing}

The harvested spines were evaluated by a 3-point bend test using a Universal Testing Machine (United STM 5802; United Testing System Inc., Fullerton, CA, USA). The spine was placed on the two fulcra with the vertebral bod- ies face down. The anvil (10 $\mathrm{mm}$ in diameter) was placed on the center of the spine above the laminectomy site, and then a load was applied with a speed of $1 \mathrm{~mm} / \mathrm{min}$. The load-displacement curves were obtained from the two experimental groups $(n=5)$ and the results were statistically compared.

\section{Histology}

For the histological analysis, hematoxylin-eosin and Masson trichrome stains of $4-\mu \mathrm{m}$-thick slices of decalcified lumbar spine were prepared to evaluate bone regeneration according to the following 10-point graded scale [8]: (1) fibrous tissue; (2) predominantly fibrous tissue with a small amount of cartilage or bone around the transverse processes; (3) equal mixture of fibrous tissue and cartilage; (4) mixed fibrous tissue and bone/cartilage; (5) some increased bone formation with a moderate fibrous gap; (6) some increased bone formation with cartilage and a small fibrous gap; (7) equal mixture of cartilage and immature bone; (8) predominantly immature bone with a small amount of cartilage; (9) union of transverse processes by immature bone; and (10) union of transverse processes by mature bone.

Histology was evaluated at 60 and 90 days postoperation (DPO), and a score of 7 or higher indicated fusion.

\section{Immunohistochemistry}

Immunohistochemical staining for osteopontin (OPN) was performed according to the conditions described in the following text. Decalcified bone slices were washed twice in phosphate-buffered saline (PBS), followed by incubation of the sections with a polyclonal OPN antibody in $1 \%$ bovine serum albumin (BSA)/PBS overnight at $4^{\circ} \mathrm{C}$. After three washes in PBS, an Alexa Fluor 488 antirabbit antibody made in goat and diluted to $1: 1,000$ in $1 \%$ BSA/PBS, was incubated with the sections for 45 minutes at room temperature. Subsequently, the sections were washed in $1 \%$ PBS and incubated with 5 drops of diamidino-2-phenylindole solution for 10 minutes. Finally, the slices were mounted with synthetic medium and covered to review OPN as a qualitative marker.

\section{Statistical analysis}

The fusion success rates between the groups were deter- 
mined by tomographic and radiographic examinations, manual palpation, and histology and were compared using the Fisher exact test. For the quantitative analysis between groups, a two-tailed Student $t$-test was performed. A probability value of $<0.05$ was considered statistically significant.

\section{Results}

All Ch/HAp scaffolds were produced using a systematic procedure and were technically evaluated by certified professionals to ensure that the described characteristics were homogeneous and under a standard constitution. We selected the 20:80 composite ratio in accordance with previous experimental data, which showed that this ratio was optimal for the promotion of bone regeneration. Lumbar experimental laminectomy and spinal fusion were performed on Wistar rats in accordance with the experimental groups, and a Ch/HAp membrane composite was used to induce bone regeneration and spinal fusion, specifically on the facet joints in the corresponding vertebrae.

\section{Lumbar fusion}

Rats treated with the Ch/HAp membrane were determined to exhibit solid fusion compared with the control group (laminectomy alone), as evaluated by tomographic examination and blinded manual palpation. Tomographic images showed a solid structure in the facet joints and intervertebral space, and this condition was considered the reference for a solid bone structure in $87 \%$ (only one with non-solid fusion and another with pseudoarthrosis) (Fig. 1). Manual palpation is a method used to evaluate spinal fusion, and if total restriction of motion is detected, spinal fusion is determined. The Ch/Hap-treated group showed no motion scores compared with the laminectomy group (Table 1).

\section{Postoperative recovery}

All rats were observed every day until 15 DPO to evaluate their postoperative recovery. A low-grade inflammatory reaction was observed at the surgical site in the membrane composite group. At 60 DPO, no signs of inflammation were seen in the Ch/HAp group, and only a small pseudocyst with non-infectious material inside was drained surgically. Normal behavior, walking, and gait of the rats were observed at 90 DPO without clinical complications.

\section{Range of motion evaluation}

The reduction in the ROM of the lumbar spine is indicative of spinal fusion. In the intact spine, the ROM was $6.96^{\circ} \pm 0.24^{\circ}$, whereas it increased and reached $20.72^{\circ} \pm 3.47^{\circ}$ in the laminectomy spine; this value was different from the ROM in the composite group, which was $7.062^{\circ} \pm 0.81^{\circ}$ (Table 1 ). The biomechanical testing analysis revealed an increment in the supported load in the dorsal space in the Ch/HA group at $90 \mathrm{DPO}$, which included the maximum load resistance until fatigue and fracture of the
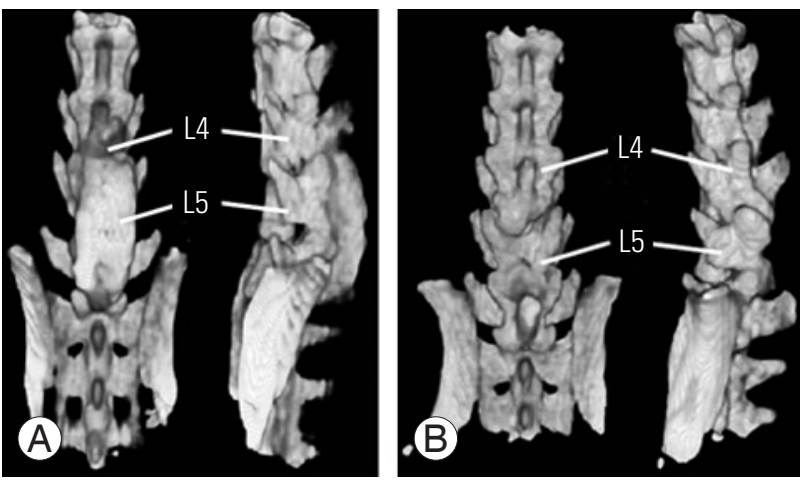

Fig. 1. Tomographic reconstruction of rat lumbar spine. The chitosanhydroxyapatite membrane group (A) had eight scores of complete trabecular fusion mass compared with no one fusion score in the control group (B). The tomographic assessment of fusion was made at 90 days postoperation. $L 4$, lumbar vertebrae 4; L5, lumbar vertebrae 5 .

Table 1. Rate of fusion through the palpation method used to evaluate spinal fusion $(n=8)$

\begin{tabular}{lcccc} 
& & & Fusion rate (\%) \\
Group & Computed tomography (\%) & Radiographic & Manual palpation & Histology \\
\cline { 3 - 5 } Control & 0 & 0 & 12.5 & 0 \\
Ch/HAp & 87 & 75 & 87 & 75 \\
\hline
\end{tabular}

The Ch/HAp-treated group showed no emotion scores compared with the laminectomy group.

$\mathrm{Ch} / \mathrm{HAp}$, chitosan-hydroxyapatite. 

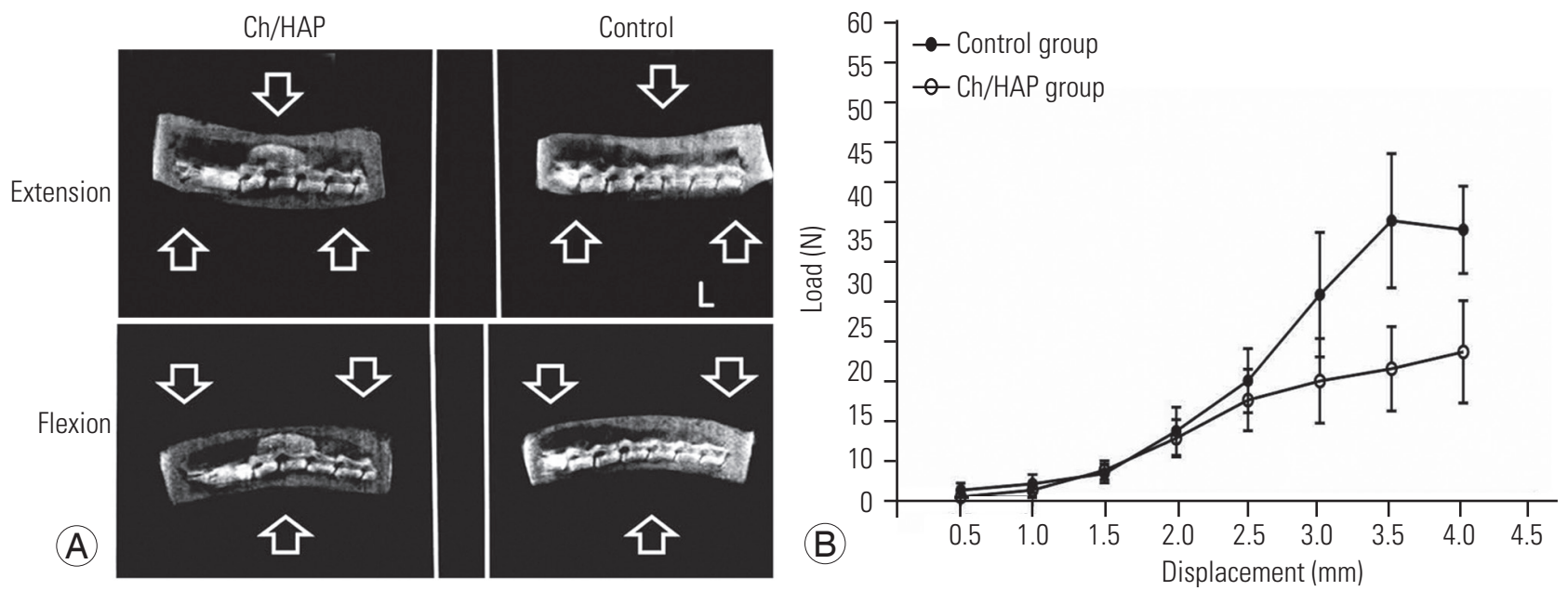

Fig. 2. Biomechanics of the rat lumbar spine. (A) Dynamic X-ray of flexion-extension movement of lumbar spine. Each spine was submitted to normal movements of the lumbar spine, and an X-ray was obtained in extension (top), and flexion (bottom). (B) Additionally, biomechanical assessment of fusion mass was evaluated through load in Newtons (N). This analysis was made at 90 days postoperation for each group. Ch/HAp, chitosanhydroxyapatite.

specimen in this group. A solid spine resists a larger load, and this robust condition was provided by the composite implant.

\section{Histological findings}

In the control group, the injured site was healed by a thin fibrotic layer that covered the entire laminectomy site (Fig. $2 \mathrm{~A}$, top). Mature bone, cartilaginous tissue, and bone remodeling units were not as evident in the control group as they were in the Ch/HAp membrane group. A low-grade inflammatory reaction, with the presence of polymorphonuclear cells, monocytes, and foreign body giant cells, was seen in the Ch/HAp membrane group, and this reaction was confirmed by histological examination at $15 \mathrm{DPO}$ (Fig. 3B).

The interface of the implant-host tissue consisting of collagen deposition was confirmed by trichrome staining, along with the presence of cartilage and new bone (Fig. 3C). The Ch/HAp membrane was eroded and was almost totally degraded at $60 \mathrm{DPO}$, and a dense fibrotic tissue replaced the wound site. This fibrotic tissue was then gradually replaced by cartilage and bone in a laminar fashion. No inflammatory cells and only a small amount of the composite material were observed at $90 \mathrm{DPO}$.

At the laminectomy site, no mature bone was observed 90 DPO in the control group by Masson trichrome staining. This differed from the Ch/HAp membrane group, in which mature bone was detected at the implant-tissue interface at $60 \mathrm{DPO}$ (Fig. 3C). Mature bone and cement lines confirmed the presence of bone in the dorsal lamina at $90 \mathrm{DPO}$ in the $\mathrm{Ch} / \mathrm{HAp}$ membrane group.

Immunohistochemical staining confirmed the expression of OPN at the bone-implant interface and even inside the matrix of the Ch/HAp membrane. OPN expression was also detected inside the attached cells on the porous surface of the composite membrane (Fig. 4).

\section{Discussion}

The aim of this study was to evaluate the efficacy of a new design for a biomaterial scaffold in a specific surgical model of experimental laminectomy. Although there have been multiple reports on HAp, there is still insufficient evidence regarding the regenerative behavior of this compound with $\mathrm{Ch}$, particularly in the specific ratio of 20\%:80\%. A membrane composite made of Ch/HAp has been synthesized by various methods and has been used in many bone defect models [11-16,19,20]. In this study, we focused on the use of this membrane as a scaffold to promote bone regeneration and to guide osteosynthesis in a laminectomy-spinal fusion model using this specific design $[21,22]$.

As a preclinical study, the main aim was to assess the biocompatibility and regenerative capacity of this compound in experimental laminectomy, compared with a group with laminectomy alone and a group of intact animals. The efficacy evaluation will be part of the continuity 

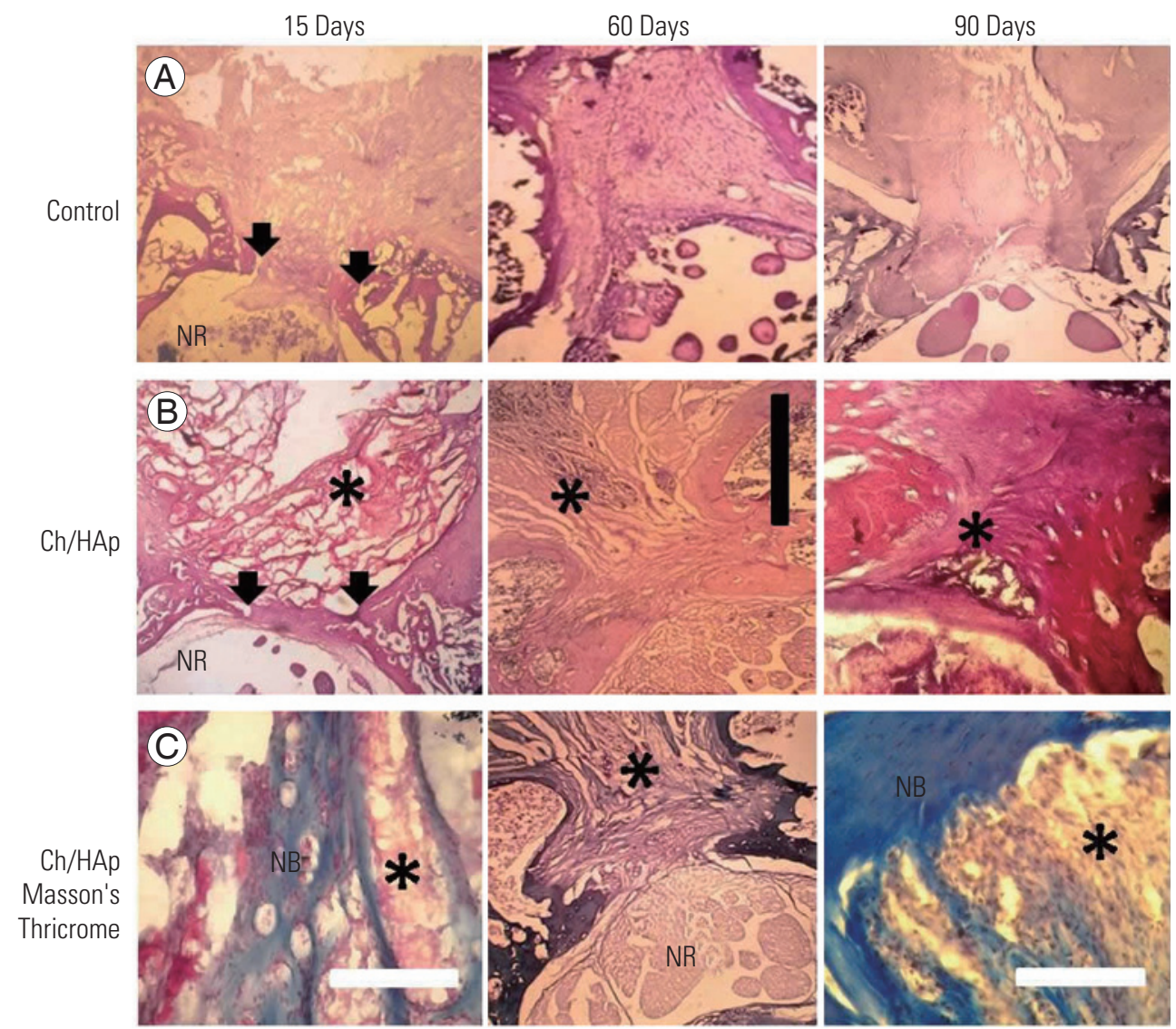

Fig. 3. Histology of rat lumbar spine. Sagittal view of 15,60 , and 90 days postoperation, respectively from left to right, of decalcified lumbar spine, the control group corresponds to the top line (A), the Ch/HAp composite group to the middle line both in H\&E stain (B). On the bottom line (C), the Ch/HAp composite group on Masson's Thricrome stain. Arrows indicate laminectomy borders of bone. Ch/HAp, chitosan-hydroxyapatite; black scale bar, 3 mm; white scale bar, $0.5 \mathrm{~mm}$; * Ch/HAp composite material; NR, nerve roots; NB, new bone.

OPN
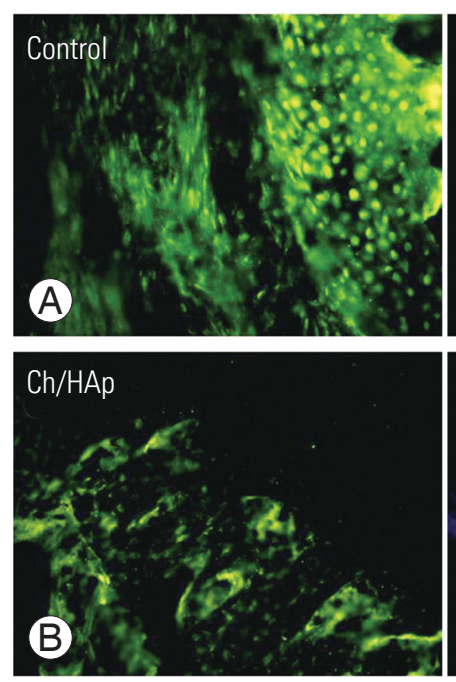

DAPI
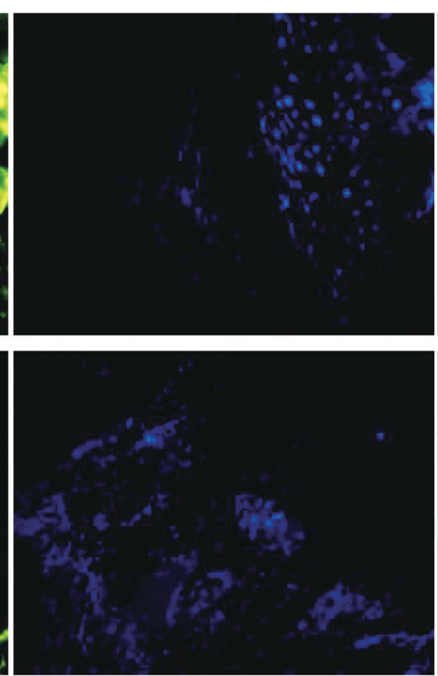

Merge
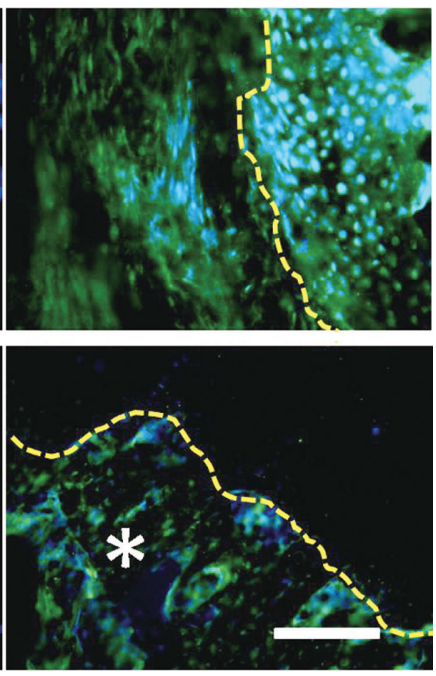

Fig. 4. OPN as a bone remodeling marker in spinal fusion. OPN expression after only laminectomy $(\mathrm{A})$ and with the $\mathrm{Ch} / \mathrm{HAp}$ composite (B). The interface between composite implant and new bone showed the most intense expression of this marker (qualitative expression). Ch/HAp, chitosan-hydroxyapatite; OPN, osteopontin; DAPI, diamidino2-pheneylindole; Merge, double immunofluorescence label; yellow dashed line, cement line (interface); ${ }^{*}, \mathrm{Ch} / \mathrm{HAp}$ composite; scale bar, $100 \mu \mathrm{m}$. 
of this study in which can compare with other standard options (gold standard) that are available as surgical materials. Lumbar fusion model with a Ch/HAp membrane as a posterolateral implant permits evaluation of the lumbar spine motion not only by manual palpation but also flexion-extension ROM, in which restriction of this range is indicative of lumbar spinal fusion compared with the intact spine group and with the control laminectomy group. The spines treated with the composite had an evidently reduced ROM compared with the control group. Our ROM analysis was based on X-ray images of the rat spine through a flexion and extension acrylic device and software as an additional contribution to this experimental model and is in agree with other studies [23].

In our study, post-laminectomy syndrome was observed in both groups (the control and Ch/HAp composite groups) and was defined as tail contracture, loss of balance, and difficulty in supporting the body with the hindlimbs. However, in the Ch/HAp implant group, this condition disappeared 30 days after surgery, suggesting the achievement of spinal stability, which was confirmed by radiological evaluation. It is important to note that this tail contracture is related more to a lack of biomechanical paravertebral muscle function and is not associated with neuropathic root damage [24].

The porosity of our composite membrane was clearly effective, because after the first 15 DPO inflammatory cells and fibroblasts were present inside the matrix, and the collagen deposition evident at 60 DPO was indicative of extracellular matrix deposition inside the implant. Our synthesized composite membrane could be a suitable scaffold for cell attachment because once it is exposed to the surrounding tissue in the surgical defect, the extracellular proteins can adhere to the membrane. This adherence would result in the establishment of an appropriate microenvironment for cell recognition, anchoring, and spreading. Porosity is an important characteristic of biocompatibility, tissue-specific induction, bio-integration, and increased mechanical strength $[22,25]$.

Bone bridging tissue formation between vertebrae is the main goal of spinal fusion. The spatial configuration of the bone mass in cases of fusion is the most important factor that leads to spinal stability and relief of pain caused by excessive spinal motion [26]. Substantial bone regeneration in the laminectomy site and additional bone formation around the facet joints of the corresponding vertebrae were induced by the Ch/HAp implant, as was accurate spinal stabilization, in our surgical model.These findings are consistent with bone regeneration, as observed in the surgical site of the defect through the extension of posterior vertebral decortications and bone ingrowth into the implant first induced by bone decortications, and also by the induction of regeneration of the surrounding soft tissue. Both processes are key to the generation of a solid structure that provides physical and biomechanical support of the lumbar spine [27].

Histological evaluation provided evidence of incremental bone regeneration and woven bone formation in the surgical defect site. Masson trichrome staining confirmed the presence of collagen deposits in the sites with the $\mathrm{Ch} /$ HAp scaffold as a part of a regenerative phase.

OPN expression is a marker of dynamic bone structure, and it is highly concentrated in preexisting and newly formed bone and on bone surfaces that interface with cells. This expression is related to the activation of osteoclasts that function in bone resorption and the consequent induction of bone formation by osteoblasts, a process termed bone remodeling. The presence of osteoclasts in the interface of the Ch/HAp membrane with bone should be the first step in bone remodeling, which occurs through the degradation of the $\mathrm{Ch}$ matrix and the liberation of calcium from the HAp. The elevation in the calcium concentration in the extracellular matrix induces osteoblast activation and bone matrix deposition inside the Ch/HAp membrane. The presence of markers in all interfaces evaluated showed an activation of the regenerative bone process, by qualitative observation, as evidence of bone formation by the implant through osteoblasts.

Epidural fibrosis, which is the most common postoperative condition in failed spine surgeries, is caused by excessive amounts of collagen deposition and tissue adherence between paravertebral muscles and nerve roots, which can also induce pain and loss of function [28,29]. As observed in this study, the Ch/HAp membrane not only prevents epidural fibrosis but also simultaneously induces bone formation of the lamina after laminectomy.

$\mathrm{Ch}$ induces neutrophil migration and the infiltration of macrophages, mast cells, and giant cells, which are common cell types that participate in the response to biomaterials implantation [30]. We found that after the implantation of the Ch/HAp composite, polymorphonuclear cells (neutrophils and macrophages) were abundant at 15 DPO, but this inflammatory reaction decreased over time, and the inflammatory tissue was replaced with non-redundant 
fibrotic tissue at $60 \mathrm{DPO}$.

The induction of hard and soft tissue regeneration in the presence of active osteoblasts and OPN expression inside the membrane composite confirms not only its good biocompatibility, but also the osteoinductive and biocompatible properties of this composite membrane.

The limitations of this study include its selective sample, and the experimental design does not include positive controls to compare to a benchmark for gold standard. The study lacks an electrophysiological evaluation, which could provide information on the position of the tail behavior and the functional pattern of gait and posture in the postoperative follow-up.

\section{Conclusions}

The Ch/HAp composite scaffold is biocompatible and induces adequate spinal stability in this spinal fusion model. In this model, the composite Ch/HAp scaffold is appropriate for bone of the lumbar spine after laminectomy and posterolateral decortication and with the surrounding tissue. Bone regeneration in a solid and well-structured fashion is induced inside the Ch/HAp composite membrane. Epidural fibrosis is limited by the physical barrier of the composite, and acceleration of the healing process is observed.

\section{Conflict of Interest}

No potential conflict of interest relevant to this article was reported.

\section{Acknowledgments}

We acknowledge National Council of Science and Technology (CONACYT), for the support provided in the "National CONACYT fellows" program, reference number: 288176. We also thank Brenda Vega- Ruíz, for their contributions in the preparation of biomaterials, and Jorge Alejandro Rochin-Mozqueda for the technical support. The authors would like to thank Enago for the English language review.

\section{References}

1. Hardman J, Graf O, Kouloumberis PE, Gao WH, Chan M, Roitberg BZ. Clinical and functional out- comes of laminoplasty and laminectomy. Neurol Res 2010;32:416-20.

2. Watkins MB. Posterolateral fusion of the lumbar and lumbosacral spine. J Bone Joint Surg Am 1953;35A:1014-8.

3. Kim DH, Jeong ST, Lee SS. Posterior lumbar interbody fusion using a unilateral single cage and a local morselized bone graft in the degenerative lumbar spine. Clin Orthop Surg 2009;1:214-21.

4. Lee JS, Suh KT. Posterior lumbar interbody fusion with an autogenous iliac crest bone graft in the treatment of pyogenic spondylodiscitis. J Bone Joint Surg Br 2006;88:765-70.

5. Logroscino CA, Proietti L, Pola E, Scaramuzzo L, Tamburrelli FC. A minimally invasive posterior lumbar interbody fusion for degenerative lumbar spine instabilities. Eur Spine J 2011;20 Suppl 1:S41-5.

6. Sasso RC, LeHuec JC, Shaffrey C; Spine Interbody Research Group. Iliac crest bone graft donor site pain after anterior lumbar interbody fusion: a prospective patient satisfaction outcome assessment. J Spinal Disord Tech 2005;18 Suppl:S77-81.

7. Summers BN, Eisenstein SM. Donor site pain from the ilium: a complication of lumbar spine fusion. J Bone Joint Surg Br 1989;71:677-80.

8. Biswas D, Bible JE, Whang PH, et al. Augmented demineralized bone matrix: a potential alternative for posterolateral lumbar spinal fusion. Am J Orthop (Belle Mead NJ) 2010;39:531-8.

9. Brandenberg G, Leibrock LG, Shuman R, Malette WG, Quigley H. Chitosan: a new topical hemostatic agent for diffuse capillary bleeding in brain tissue. Neurosurgery 1984;15:9-13.

10. Lee M, Li W, Siu RK, et al. Biomimetic apatite-coated alginate/chitosan microparticles as osteogenic protein carriers. Biomaterials 2009;30:6094-101.

11. Shi C, Zhu Y, Ran X, Wang M, Su Y, Cheng T. Therapeutic potential of chitosan and its derivatives in regenerative medicine. J Surg Res 2006;133:185-92.

12. Hing KA, Best SM, Tanner KE, Bonfield W, Revell PA. Mediation of bone ingrowth in porous hydroxyapatite bone graft substitutes. J Biomed Mater Res A 2004;68:187-200.

13. Cuenca-Lopez MD, Andrades JA, Gomez S, et al. Evaluation of posterolateral lumbar fusion in sheep using mineral scaffolds seeded with cultured bone marrow cells. Int J Mol Sci 2014;15:23359-76. 
14. Ding M, Koroma KE, Sorensen JR, et al. Collagenhydroxyapatite composite substitute and bone marrow nuclear cells on posterolateral spine fusion in sheep. J Biomater Appl 2019;34:365-74.

15. Yoshida A, Miyazaki T, Ishida E, Ashizuka M. Preparation of bioactive chitosan-hydroxyapatite nanocomposites for bone repair through mechanochemical reaction. Mater Trans 2004;45:994-8.

16. Zo SM, Singh D, Kumar A, Cho YW, Oh TH, Han SS. Chitosan-hydroxyapatite macroporous matrix for bone tissue engineering. Curr Sci 2012;103:1438-46.

17. Lenke LG, Bridwell KH, Bullis D, Betz RR, Baldus C, Schoenecker PL. Results of in situ fusion for isthmic spondylolisthesis. J Spinal Disord 1992;5:433-42.

18. Dimar JR 2nd, Ante WA, Zhang YP, Glassman SD. The effects of nonsteroidal anti-inflammatory drugs on posterior spinal fusions in the rat. Spine (Phila Pa 1976) 1996;21:1870-6.

19. Lee JS, Baek SD, Venkatesan J, et al. In vivo study of chitosan-natural nano hydroxyapatite scaffolds for bone tissue regeneration. Int J Biol Macromol 2014;67:360-6.

20. Yuan H, Chen N, Lu X, Zheng B. Experimental study of natural hydroxyapatite/chitosan composite on reconstructing bone defects. J Nanjing Med Univ 2008;22:372-5.

21. Rodriguez-Vazquez M, Vega-Ruiz B, Ramos-Zuniga R, Saldana-Koppel DA, Quinones-Olvera LF. Chitosan and its potential use as a scaffold for tissue engineering in regenerative medicine. Biomed Res Int 2015;2015:821279.

22. Vega-Ruiz B, Ramos-Zuniga R, Duran IS, UrsielOrtega Y. Biomaterials and surgical applications: the translational perspective. Transl Surg 2017;2:85-102.
23. Yamaguchi T, Inoue N, Sah RL, et al. Micro-computed tomography-based three-dimensional kinematic analysis during lateral bending for spinal fusion assessment in a rat posterolateral lumbar fusion model. Tissue Eng Part C Methods 2014;20:578-87.

24. Massie JB, Huang B, Malkmus S, et al. A preclinical post laminectomy rat model mimics the human post laminectomy syndrome. J Neurosci Methods 2004;137:283-9.

25. Zhang J, Liu G, Wu Q, Zuo J, Qin Y, Wang J. Novel mesoporous hydroxyapatite/chitosan composite for bone repair. J Bionic Eng 2012;9:243-51.

26. Shin DA, Yang BM, Tae G, Kim YH, Kim HS, Kim HI. Enhanced spinal fusion using a biodegradable porous mesh container in a rat posterolateral spinal fusion model. Spine J 2014;14:408-15.

27. Cunningham BW, Shimamoto N, Sefter JC, et al. Osseointegration of autograft versus osteogenic protein-1 in posterolateral spinal arthrodesis: emphasis on the comparative mechanisms of bone induction. Spine J 2002;2:11-24.

28. Seyedmajidi M, Rabiee S, Haghanifar S, et al. Histopathological, histomorphometrical, and radiographical evaluation of injectable glass-ceramic-chitosan nanocomposite in bone reconstruction of rat. Int J Biomater 2015;2015:719574.

29. Carvalho M, Costa LM, Pereira JE, et al. The role of hybrid chitosan membranes on scarring process following lumbar surgery: post-laminectomy experimental model. Neurol Res 2015;37:23-9.

30. Farrugia BL, Whitelock JM, Jung M, et al. The localisation of inflammatory cells and expression of associated proteoglycans in response to implanted chitosan. Biomaterials 2014;35:1462-77. 